\section{Lumacaftor/ivacaftor for patients homozygous for Phe508del-CFTR: should we curb our enthusiasm?}

\author{
Andrew M Jones, Peter J Barry
}

Cystic fibrosis (CF) is a success story of modern medicine, with advances in care transforming a disease previously associated with early childhood death into a chronic condition with a predicted median survival of nearly 50 years for patients born in this current decade. ${ }^{1}$ This has been achieved through a model of multidisciplinary care with the successive advent of supportive therapies that tackled the consequences of the condition, such as replacement pancreatic enzymes, inhaled antibiotics and agents to improve mucociliary clearance. The quest for treatments that address the underlying basic defect has been a hope for the CF community since the discovery of the CF gene, which codes for the dysfunctional channel protein, the CF transmembrane conductance regulator (CFTR), in $1989 .^{2}$ The Cystic Fibrosis Foundation supported an ambitious pipeline to identify molecules that could allow correction of the dysfunctional CFTR protein, which began to bear the fruits of investment with the treatment of CF patients with Gly551Asp (G551D) mutation with ivacaftor.

The Gly551Asp mutation produces a CFTR protein that localises to the epithelial cell membrane but fails to open. Ivacaftor acts as a CFTR potentiator that increases channel opening probability and has demonstrated remarkable improvements in lung function, nutritional status, patient-reported outcome measures and biomarkers of CFTR function in clinical studies of patients with the Gly551Asp gene mutation. ${ }^{3}{ }^{4}$ The initial promise of these significant clinical benefits seen in pre-licensing clinical studies has been fulfilled in subsequent postmarketing phase IV studies. ${ }^{5-7}$ In addition, published case reports and anecdotal comments from patients have exemplified the benefits of such therapy beyond the respiratory

Manchester Adult Cystic Fibrosis Centre, University Hospital of South Manchester NHS Trust, Manchester, UK

Correspondence to Dr Andrew M Jones, Manchester Adult Cystic Fibrosis Centre, University Hospitals South Manchester NHS Trust, Southmoor Road,

Wythenshawe, Manchester M23 9LT, UK; andrew.jones@uhsm.nhs.uk system in addressing the dysfunction that CF causes in other organs and systems. ${ }^{8-10}$

The unprecedented success of ivacaftor treatment for the Gly551Asp CF patient population has created immense excitement within the CF community with anticipation of similar therapies becoming available for the remainder of patients with other CF gene mutations. The Gly551Asp mutation is encountered in approximately $5 \%$ of patients, while Phe508del is by far the most common, accounting for approximately $70 \%$ of the CF mutations worldwide, positioning it as the major target for new treatments.

The Phe508del mutation causes abnormal folding and trafficking of CFTR to the epithelial cell membrane, and also abnormal opening of the channel in the limited amounts of protein that make it to the cell surface. Wainwright and colleagues recently published the results of two large international multicentre phase III studies (TRAFFIC and TRANSPORT) of a combination of two small moleculeslumacaftor, a CFTR corrector that targets the folding deficiency, and ivacaftor-in patients homozygous for Phe508del. ${ }^{11} \mathrm{~A}$ total of 1108 patients were randomised $(1: 1: 1)$ to lumacaftor $600 \mathrm{mg}$ daily/ivacaftor $250 \mathrm{mg}$ twice daily, lumacaftor $400 \mathrm{mg}$ twice daily/ivacaftor $250 \mathrm{mg}$ twice daily, or placebo. The study achieved its primary endpoint of a mean absolute change from baseline of $\% \mathrm{FEV}_{1}$ of $2.6-4 \%$ in each of the combination treatment groups in both studies, in comparison to placebo at 24 weeks $(p<0.001)$. In pooled analyses of both studies, pulmonary exacerbations were $30 \%$ lower in the $600 \mathrm{mg} /$ day of lumacaftor group $(\mathrm{p}=0.001)$ and $39 \%$ lower in the $400 \mathrm{mg} / 12 \mathrm{~h}$ of lumacaftor group compared with placebo $(\mathrm{p}<0.001)$.

The question of whether these results merit the heralding of the study as a landmark advance in CF therapeutics is, however, open to debate. With approximately $45 \%$ of patients with CF homozygous for the Phe508del mutation, has the fulfilment of promise of effective CFTR modulation therapy for the largest cohort of patients been delivered? That this study represents a potential advance for CF therapeutics is not in doubt. The trial design, in particular, should be commended on the large numbers recruited (which in fact do represent a landmark for CF trials) and the permitted use of standard CF therapies for all participants, a factor often overlooked in other trials. However, the improvements in lung function seen in the study are modest at best, and considerably less than those for ivacaftor in patients with Gly551Asp mutation. ${ }^{3}{ }^{4}$ This lack of enthusiasm does not solely represent an elevated sense of expectation following the initial experience with ivacaftor as the improvements in $\% \mathrm{FEV}_{1}$ are similar to those previously witnessed for a number of other treatments currently available that address the downstream consequences of CFTR dysfunction, such as DNAase, azithromycin and nebulised antibiotics. ${ }^{12-14}$ Similarly, the reduction in pulmonary exacerbations is not an outlier when grouped with other pre-modulation therapies, a fact even more notable given the relatively low uptake of chronic azithromycin therapy in this cohort (61.4\%).

The need for two molecules working on the processing and functioning of abnormal CFTR presents its own challenges. The potential for adverse events and drugdrug interactions will always increase with polypharmacy. The combination product was generally well tolerated in this study with low rates of discontinuations. Early chest tightness was witnessed in the treatment arm mimicking results of the phase II study. Although minor elevations of transaminases were seen across all groups in the study, more significant elevations of levels to three times the upper limit of normal in association with bilirubin elevations to twice the upper limit of normal, although rare, were only encountered in patients taking LUM/IVA (three patients). There is pharmacokinetic evidence of interaction between the two compounds in question, explaining the higher doses of ivacaftor used. There is also in vitro evidence of an interaction, with prolonged use of potentiators decreasing the stability of 'corrected' Phe508del CFTR. ${ }^{15}{ }^{16}$ Could this finding explain the relatively modest improvement in pulmonary function? It almost certainly suggests the need for a further additive therapy with the aim of stabilising corrected CFTR.

The absence of data on sweat chloride levels or other CFTR biomarker appears an obvious omission. The changes in these parameters were modest in the phase II trials $(-8.9$ to $-10.3 \mathrm{mmol} / \mathrm{L})$, and while sweat chloride levels do not correlate with clinical response to ivacaftor in patients with Gly551Asp mutation, they still may represent a biomarker of CFTR activity. ${ }^{17-}$ 
19 We can infer from this that the restoration of CFTR function in this population is certainly less profound than what we have previously witnessed. The phase II study of this combination product could not establish a benefit for Phe508del heterozygotes, suggesting that what we are witnessing is low-level CFTR modulation that requires the presence of two suitable alleles to detect clinical effects. We will await further reports on whether this low-level modulation is sufficient to impact on the multisystem nature of the disease. The improvements in nutritional status were small and did not reach significance in all treatment arms of the study. Similarly, the improvements in patientreported outcome measures reached statistical significance but not at the accepted level of clinical significance.

The US Food and Drug Administration (FDA) Pulmonary-Allergy Drugs Advisory Committee recently voted 12 to 1 in favour of a recommendation for the use of the combination of lumacaftor/ivacaftor product (Orkambi) in patients with $\mathrm{CF}$ ages 12 and older who are homozygous for the F508del mutation; a final FDA decision on licensing for Orkambi is expected in July 2015. The success of these and previous studies has boosted the share price of Vertex Pharmaceuticals and delivered great awards for investors, including the Cystic Fibrosis Foundation, which recently sold its investment for $>\$ 3.3$ billion. Many members of the CF community have contributed their time to studies of CFTR therapy and deserve access to an affordable product that reflects the gains. Affordability is a key issue. The costing of ivacaftor for patients with Gly551Asp mutation delayed its introduction in many countries. The reimbursement price for ivacaftor is purported to represent both the development cost and its limitation to a small target population of CF sufferers (approximately 5\%). The pricing of Orkambi should reflect the nine times larger target patient population, together with the much more modest clinical response. Would this in turn have a downstream effect on the costing of ivacaftor monotherapy? If not, we are in a baffling economic situation whereby monotherapy could be more expensive than a combination product that includes greater quantities of ivacaftor and an 'added extra'! This added extra may even provide the possibility to further improve symptoms for Phe508del/ Gly551Asp patients! $!^{20}$
The drug pipeline continues with the recent opening of phase III clinical trials with a further CFTR corrector VX-661; however, the results of phase II studies with this combination were not substantially better. ${ }^{21}$ To focus on just these compounds also fails to address the needs of other patients with CF with rarer genotypes. In tandem with drug development needs to be the development of superior patient-specific biomarkers that may be able to predict responses to therapies ex vivo permitting those patients to gain access to these medications.

It should be recognised that even in the best representation of effective CFTR modulation, ivacaftor in patients with Gly551Asp mutation, adherence has been reported to be suboptimal and responses can be heterogeneous. These factors will need to be considered should combination therapy receive licensing, and effectiveness will need to be explored in a real-world setting, where lung function responses may not be as great as those seen in clinical trials. The results of this study represent a success for the CF community and should be welcomed as such; however, the recognition that these results should not represent the 'holy grail' for Phe508del homozygote patients is equally important.

Competing interests AMJ has previously received consultancy fees from Gilead Sciences and Vertex Pharmaceuticals, and support for educational meetings from Forrest. PJB has received consultancy fees from Vertex Pharmaceuticals.

Provenance and peer review Commissioned; internally peer reviewed.

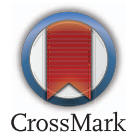

To cite Jones AM, Barry PJ. Thorax 2015;70:615616.

Received 1 June 2015

Accepted 2 June 2015

Thorax 2015;70:615-616.

doi:10.1136/thoraxjnl-2015-207369

\section{REFERENCES}

1 Dodge JA, Lewis PA, Stanton M, et al. Cystic fibrosis mortality and survival in the UK: 1947-2003. Eur Respir J 2007:29:522-6.

2 Riordan JR, Rommens JM, Kerem B, et al. Identification of the cystic fibrosis gene: cloning and characterization of complementary DNA. Science 1989:245:1066-73.

3 Ramsey BW, Davies J, McElvaney NG, et al. A CFTR potentiator in patients with cystic fibrosis and the G551D mutation. N Eng/ J Med 2011;365:1663-72.

4 Davies JC, Wainwright CE, Canny GJ, et al. Efficacy and safety of ivacaftor in patients aged 6 to 11 years with cystic fibrosis with a G551D mutation. Am J Respir Crit Care Med 2013;187:1219-25.
5 Barry PJ, Plant BJ, Nair A, et al. Effects of ivacaftor in patients with cystic fibrosis who carry the G551D mutation and have severe lung disease. Chest 2014:146:152-8.

6 McKone EF, Borowitz D, Drevinek $P$, et al, VX08-770-105 (PERSIST) Study Group. Long-term safety and efficacy of ivacaftor in patients with cystic fibrosis who have the Gly551Asp-CFTR mutation: a phase 3, open-label extension study (PERSIST). Lancet Respir Med 2014;2:902-10.

7 Rowe SM, Heltshe SL, Gonska T, et al. Clinical mechanism of the cystic fibrosis transmembrane conductance regulator potentiator ivacaftor in G551D-mediated cystic fibrosis. Am J Respir Crit Care Med 2014;90:175-84

8 Hayes D Jr, Warren PS, McCoy KS, et al. Improvement of hepatic steatosis in cystic fibrosis with ivacaftor therapy. J Pediatr Gastroenterol Nutr 2015;60:578-9.

9 Grasemann H, Ratjen F, Solomon M. Aquagenic wrinkling of the palms in a patient with cystic fibrosis. N Engl J Med 2013;369:2362-3.

10 Bellin MD, Laguna T, Leschyshyn J, et al. Insulin secretion improves in cystic fibrosis following ivacaftor correction of CFTR: a small pilot study. Pediatr Diabetes 2013;14:417-21.

11 Wainwright CE, Elborn JS, Ramsey BW, et al. Lumacaftor-ivacaftor in patients with cystic fibrosis homozygous for Phe508del CFTR. N Engl J Med. Published Online First: 17 May 2015. doi:10.1056/ NEJMoa1409547.

12 Fuchs HJ, Borowitz DS, Christiansen DH, et al. Effect of recombinant human DNase on exacerbations of respiratory symptoms and on pulmonary function in patients with cystic fibrosis. N Engl J Med 1994;331:637-42.

13 Saiman L, Marshall BC, Mayer-Hamblett N, et al. Azithromycin in patients with cystic fibrosis chronically infected with Pseudomonas aeruginosa A randomized controlled trial. J Am Med Assoc 2003;290:1749-56.

14 Ramsey BW, Pepe MS, Quan JM, et al. Intermittent administration of inhaled tobramycin in patients with cystic fibrosis. N Engl J Med 1999;340:23-30.

15 Veit G, Avramescu RG, Perdomo D, et al. Some gating potentiators, including VX-770, diminish $\Delta$ F508-CFTR functional expression. Sci Trans/ Med 2014;6:246ra97.

16 Cholon DM, Quinney NL, Fulcher ML, et al. Potentiator ivacaftor abrogates pharmacological correction of $\Delta$ F508 CFTR in cystic fibrosis. Sci Transl Med 2014;6:246ra96.

17 Boyle MP, Bell SC, Konstan MW, et al, VX09-809102 study group. A CFTR corrector (lumacaftor) and a CFTR potentiator (ivacaftor) for treatment of patients with cystic fibrosis who have a phe508del CFTR mutation: a phase 2 randomised controlled trial. Lancet Respir Med 2014:2:527-38.

18 Barry PJ, Jones AM, Webb AK, et al. Sweat chloride is not a useful marker of clinical response to Ivacaftor. Thorax 2014;69:586-7.

19 Accurso FJ, Van Goor F, Zha J, et al. Sweat chloride as a biomarker of CFTR activity: proof of concept and ivacaftor clinical trial data. J Cyst Fibros 2014;13:139-47.

20 Donaldson SH, Pilewski JM, Cooke J, et al. Addition of VX-661, an investigational CFTR corrector, to ivacaftor, a CFTR potentiator, in patients with CF and heterozygous for F508del/G551D-CFTR. Pediatr Pulmonol 2014:49(S38):S308-9.

21 Donaldson S, Pilewski J, Griese M, et al. WS7. 3 VX-661, an investigational CFTR corrector, in combination with ivacaftor, a CFTR potentiator, in patients with CF and homozygous for the F508DelCFTR mutation: Interim analysis. J Cyst Fibros 2013;12(Suppl 1):S1-160. 